\title{
The First Simultaneous X-Ray/Radio Detection of the First Be/BH System MWC 656
}

\author{
M. Ribó ${ }^{1,11}$, P. Munar-Adrover ${ }^{2}$, J. M. Paredes ${ }^{1}$, B. Marcote ${ }^{1,3}$, K. Iwasawa ${ }^{4}$, J. Moldón ${ }^{1,5,6}$, J. Casares ${ }^{7,8,9}$, \\ S. Migliari $^{10}$, and X. Paredes-Fortuny ${ }^{1}$ \\ ${ }^{1}$ Departament de Física Quàntica i Astrofísica, Institut de Ciències del Cosmos (ICCUB), Universitat de Barcelona, IEEC-UB, \\ Martí i Franquès 1, E08028 Barcelona, Spain \\ ${ }^{2}$ INAF/IAPS-Roma, I-00133 Roma, Italy \\ 3 Joint Institute for VLBI ERIC (JIVE), Postbus 2, 7990 AA Dwingeloo, The Netherlands \\ ${ }_{5}^{4}$ ICREA, Institut de Ciències del Cosmos (ICCUB), Universitat de Barcelona, IEEC-UB, Martí i Franquès 1, E-08028 Barcelona, Spain \\ 5 Jodrell Bank Center for Astrophysics, School of Physics and Astronomy, The University of Manchester, Manchester M13 9PL, UK \\ ${ }^{6}$ ASTRON, The Netherlands Institute for Radio Astronomy, Postbus 2, 7990 AA, Dwingeloo, The Netherlands \\ 7 Instituto de Astrofísica de Canarias, E-38200 La Laguna, Tenerife, Spain \\ ${ }^{8}$ Departamento de Astrofísica, Universidad de La Laguna, Avda. Astrofísico Francisco Sánchez s/n, E-38271 La Laguna, Tenerife, Spain \\ ${ }_{9}$ Department of Physics, Astrophysics, University of Oxford, Denys Wilkinson Building, Keble Road, Oxford OX1 3RH, UK \\ ${ }^{10}$ European Space Astronomy Centre, Apartado/P.O. Box 78, Villanueva de la Canada, E-28691 Madrid, Spain \\ Received 2016 November 6; revised 2017 January 9; accepted 2017 January 10; published 2017 February 1
}

\begin{abstract}
MWC 656 is the first known Be/black hole (BH) binary system. Be/BH binaries are important in the context of binary system evolution and sources of detectable gravitational waves because they are possible precursors of coalescing neutron star/BH binaries. X-ray observations conducted in 2013 revealed that MWC 656 is a quiescent high-mass X-ray binary (HMXB), opening the possibility to explore X-ray/radio correlations and the accretion/ ejection coupling down to low luminosities for BH HMXBs. Here we report on a deep joint Chandra/VLA observation of MWC 656 (and contemporaneous optical data) conducted in 2015 July that has allowed us to unambiguously identify the X-ray counterpart of the source. The X-ray spectrum can be fitted with a power law with $\Gamma \sim 2$, providing a flux of $\simeq 4 \times 10^{-15} \mathrm{erg} \mathrm{cm}^{-2} \mathrm{~s}^{-1}$ in the $0.5-8 \mathrm{keV}$ energy range and a luminosity of $L_{\mathrm{X}} \simeq 3 \times 10^{30} \mathrm{erg} \mathrm{s}^{-1}$ at a $2.6 \mathrm{kpc}$ distance. For a $5 \mathrm{M}_{\odot}$ BH this translates into $\simeq 5 \times 10^{-9} L_{\text {Edd. }}$. These results imply that MWC 656 is about 7 times fainter in X-rays than it was two years before and reaches the faintest X-ray luminosities ever detected in stellar-mass BHs. The radio data provide a detection with a peak flux density of $3.5 \pm 1.1 \mu \mathrm{Jy}$ beam $^{-1}$. The obtained X-ray/radio luminosities for this quiescent BH HMXB are fully compatible with those of the X-ray/radio correlations derived from quiescent BH low-mass X-ray binaries. These results show that the accretion/ejection coupling in stellar-mass BHs is independent of the nature of the donor star.
\end{abstract}

Key words: binaries: general - black hole physics - stars: emission-line, Be - stars: individual (MWC 656) X-rays: binaries - X-rays: individual (MWC 656)

\section{Introduction}

X-ray observations of stellar-mass black holes (BHs) in binary systems have allowed for the study of the properties of the accretion disks that surround them, as well as their changes over time (see Remillard \& McClintock 2006 for a review). Radio observations of these systems have allowed us to gain knowledge of the ejection processes in the form of relativistic jets (Mirabel \& Rodríguez 1999; Fender 2001; Fender \& Muñoz-Darias 2016). Simultaneous observations have revealed the existence of non-linear correlations between the X-ray and radio luminosities during the so-called low/hard and quiescent states that are consistent with scale-invariant jet models (Corbel et al. 2003; Gallo et al. 2003; Markoff et al. 2003; Fender et al. 2004; Fender \& Belloni 2004; Corbel et al. 2013; Gallo et al. 2014). However, these studies have mainly been conducted for $\mathrm{BHs}$ in low-mass X-ray binaries (LMXBs) because the only confirmed $\mathrm{BH}$ in a high-mass X-ray binary (HMXB) in the Galaxy before the discovery of MWC 656, namely Cygnus X-1, always displays a high luminosity and does not allow us to trace the correlation (see, e.g., Zdziarski 2012).

MWC 656 is the first binary system containing a BH in orbit around a Be star (Casares et al. 2014). Its discovery was

\footnotetext{
${ }^{11}$ Serra Húnter Fellow.
}

triggered by the detection, with AGILE, of the transient gamma-ray source AGL J2241+4454 (Lucarelli et al. 2010), undetected with Fermi/LAT (Alexander \& McSwain 2015) but recently reported to show recurrent activity with hints of longterm variability with AGILE (Munar-Adrover et al. 2016). The binary nature of MWC 656 (a bright Be star also named HD 215227) was suggested by a 60.37 d periodicity in optical photometry (Williams et al. 2010; Paredes-Fortuny et al. 2012), and later confirmed through radial velocity studies (Casares et al. 2012). A detailed spectroscopic analysis revealed the presence of a He II emission line at $\lambda 4686 \AA$ that could only be produced in an accretion disk around the invisible companion to the Be star. A re-analysis of radial velocity data using an Fe II emission line from the Be circumstellar disk and the He II emission line from the accretion disk, together with an improved spectral type classification of B1.5-B2 III for the Be star, provided a mass for the compact object in the range of 3.8-6.9 $\mathrm{M}_{\odot}$, thus confirming its $\mathrm{BH}$ nature (Casares et al. 2014).

The discovery of the first $\mathrm{Be} / \mathrm{BH}$ system is relevant in the context of binary system evolution. Although it partially solves the problem of the absence of $\mathrm{Be} / \mathrm{BH}$ systems (Belczynski \& Ziolkowski 2009), the discovery of MWC 656 is observationally biased by the lack of a bright X-ray counterpart, suggesting that there might be a large number of hidden $\mathrm{Be} / \mathrm{BH}$ systems. 
In addition, simulations have shown that it is very difficult to form $\mathrm{Be} / \mathrm{BH}$ binaries with properties similar to those of MWC 656, although they can evolve into close NS/BH systems that will merge on timescales of a few Gyr and produce gravitational waves detectable with advanced LIGO/ Virgo in nearby galaxies (Grudzinska et al. 2015).

Munar-Adrover et al. (2014) conducted a 14-ks XMMNewton observation of MWC 656 on 2013 June 4 that provided the detection between 0.3 and $5.5 \mathrm{keV}$ of a faint source coincident at the $2.4 \sigma$ level with MWC 656. This revealed that MWC 656 was a new HMXB. The spectrum analysis required a model fit with two components, a blackbody plus a power law, the latter dominating above $\simeq 0.8 \mathrm{keV}$. The thermal emission was proposed to arise from the wind of the $\mathrm{Be}$ star, while the non-thermal emission arose from the vicinity of the $\mathrm{BH}$. The observed non-thermal luminosity was $L_{\mathrm{X}}=\left(1.6_{-0.9}^{+1.0}\right) \times 10^{31} \mathrm{erg} \mathrm{s}^{-1}$, or $(3.1 \pm 2.3) \times 10^{-8} L_{\mathrm{Edd}}$ for a source distance of $2.6 \pm 0.6 \mathrm{kpc}$, indicating a $\mathrm{BH}$ in deep quiescence (see Plotkin et al. 2013 for reference).

Radio observations with different interferometers, frequencies, and epochs have provided $3 \sigma$ upper limits to the flux density as low as $30 \mu \mathrm{Jy}$ (Moldón 2012; Marcote 2015). Recently, Dzib et al. (2015) have reported a detection with VLA at a peak flux density of $10 \pm 3 \mu \mathrm{Jy}$ beam $^{-1}$. Observations at $\mathrm{TeV}$ energies with the MAGIC telescopes have only led to upper limits (Aleksić et al. 2015). For a detailed review on MWC 656 see Ribó 2015.

In this Letter, we report on a deep joint Chandra/VLA observation (and contemporaneous optical data) of the first Be/ BH binary MWC 656 that has allowed us to: 1) resolve the XMM-Newton detection into two sources, one of them unambiguously associated with MWC 656;2) find that it is significantly fainter than two years before and reaches the faintest quiescent luminosities detected in stellar-mass BHs; and 3 ) detect a faint radio counterpart and find that the obtained $\mathrm{X}$-ray/radio luminosities for this quiescent $\mathrm{BH} \mathrm{HMXB}$ are fully compatible with those of the $\mathrm{X}$-ray/radio correlations derived from quiescent $\mathrm{BH}$ LMXBs at the low-luminosity end.

\section{Observations, Data Analysis, and Results}

\subsection{X-rays}

MWC 656 was observed with the Chandra X-ray Observatory ACIS-S camera using the VFAINT data mode during $\sim 60 \mathrm{ks}$, from 2015 July 24 at 21:03 UT to July 25 at 14:29 UT (PI: M. Ribó; Chandra ObsId 16753 ). The observation covered the orbital phase range 0.01-0.02 (using $\mathrm{JD}_{0}=2453243.3$ from Williams et al. 2010). The data were analyzed using the Chandra Interactive Analysis of Observations (CIAO) software, v4.7 ${ }^{12}$ (Fruscione et al. 2006). The event files were reprocessed by means of the chandra_repro script. No flares were detected in the background light curve, yielding an effective exposure time of $59.1 \mathrm{ks}$. The data analysis was performed in the $0.5-8 \mathrm{keV}$ energy band at which Chandra presents higher sensitivity.

We show the Chandra X-ray image of the field around MWC 656 in Figure 1, together with the contours of the previous XMM-Newton image reported in Munar-Adrover et al. (2014). Inspection of the X-ray image superimposing optical positions from the USNO-B1.0 catalog (Monet

\footnotetext{
12 http://cxc.harvard.edu/ciao/index.html
}

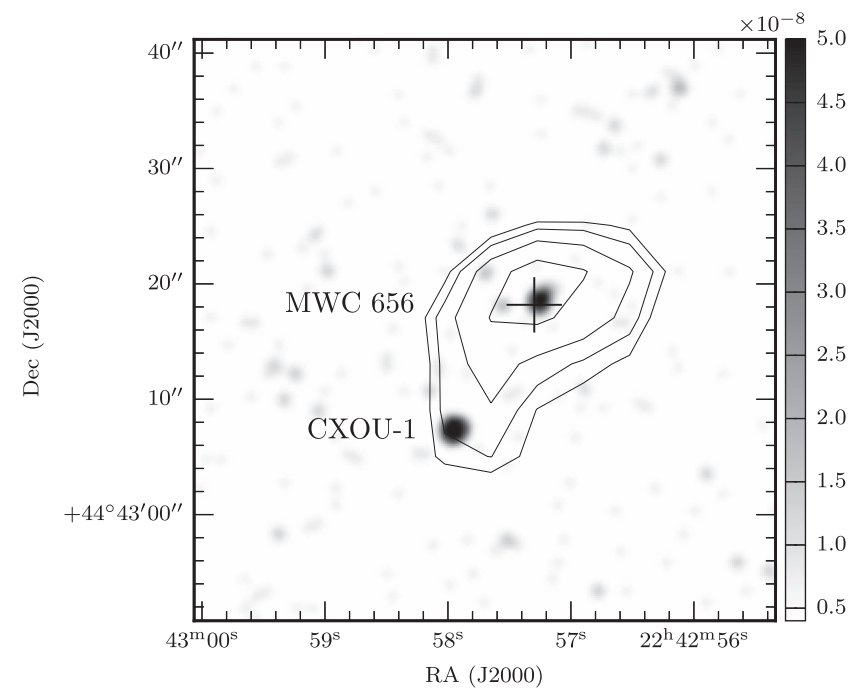

Figure 1. Chandra ACIS-S image of $50^{\prime \prime} \times 50^{\prime \prime}$ at the position of MWC 656 (indicated by the cross) in the $0.5-8.0 \mathrm{keV}$ energy band smoothed using a Gaussian interpolation with a $3^{\prime \prime}$ kernel. The flux scale appears on the right of the image. Contours correspond to the previous XMM-Newton EPIC-pn image from Munar-Adrover et al. (2014).

et al. 2003) reveals a systematic offset. Using three common sources we have applied a correction of R.A. $=+0 . .5$ and decl. $=+0$ ". 1 to the Chandra data (and of R.A. $=+2$ ". 4 and decl. $=+4.9$ to the XMM-Newton data using the only common source). The better angular resolution of Chandra has allowed us to resolve the XMM-Newton source into two sources. We have used the wavdetect algorithm to obtain the significance and positions of both sources. MWC 656 is detected at the $8.5 \sigma$ confidence level (c.l.), with equatorial coordinates (after correction) R.A. $=22^{\mathrm{h}} 42^{\mathrm{m}} 57^{\mathrm{s}} .27 \pm 0.01$, decl. $=44^{\circ} 43^{\prime} 18^{\prime \prime} .5 \pm 0$." 1 (uncertainties are statistical only and at $1 \sigma$ c.1.). The position of MWC 656 from Gaia data at the epoch of the Chandra observation $($ epoch $=2015.56$ ) following Gaia Collaboration et al. (2016) and Gaia Collaboration (2016) is: R.A. $=22^{\mathrm{h}} 42^{\mathrm{m}} 57^{\mathrm{s}} .29718 \pm$ 0.00002, decl. $=44^{\circ} 43^{\prime} 18$ " $2099 \pm 0$ " 0002 . The offset between both positions is 0 ". $4 \pm 0$ ". $1_{\text {stat }} \pm 0$ ! $1_{\text {syst }}$, and the Chandra source is thus the counterpart of MWC 656.

The new source, located $\sim 13 . " 5$ to the southeast of MWC 656, is detected at a $15.9 \sigma$ c.l. and has equatorial coordinates (after correction) R.A. $=22^{\mathrm{h}} 42^{\mathrm{m}} 57^{\mathrm{s}} .969 \pm 0.005$, decl. $=44^{\circ} 43^{\prime} 07 . \prime 34 \pm 0 !^{\prime \prime} 05$, and thus is named CXOU J224257.9+444307 (CXOU-1 from now on). The Chandra image reveals that MWC 656 is fainter than CXOU-1 (see below), while the shape of the XMM-Newton contours reveals that in 2013 June MWC 656 was brighter than CXOU-1.

With the use of the srcflux script we extracted net counts for MWC $656 \quad(22.1 \pm 4.9$ at $90 \%$ c.l. $)$ and CXOU-1 $(38.7 \pm 6.3)$, taking into account the background and the point-spread function of the instrument. These measurements were obtained from a region centered at the peak of the X-ray emission (found by wavdetect) of each source with a radius of $3^{\prime \prime}$, while background counts were obtained from a nearby circular region with a $10^{\prime \prime}$ radius, avoiding contamination from nearby sources, always within the $0.5-8.0 \mathrm{keV}$ energy band.

We used XSPEC version 12.8.2 (Arnaud 1996) to perform a spectral analysis of both sources. The same regions for source and background used to extract net counts were considered. We 

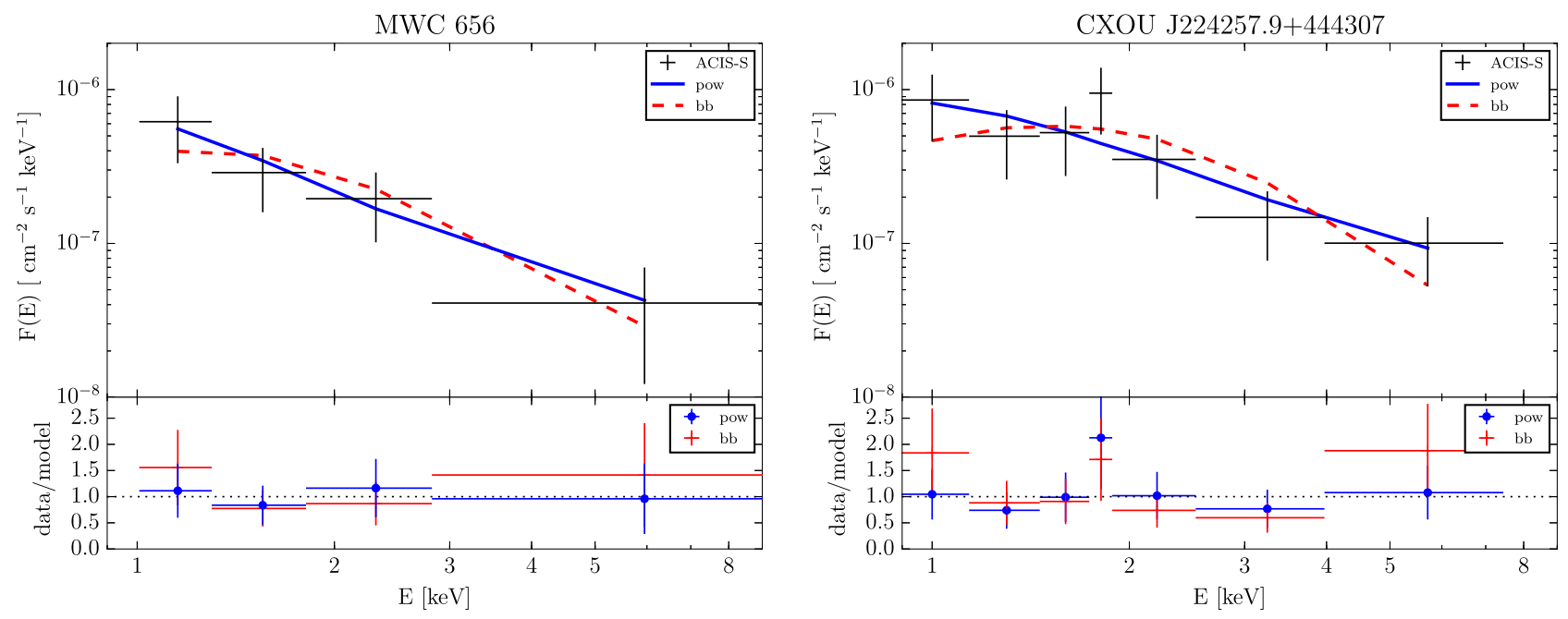

Figure 2. Left: MWC 656 Chandra ACIS-S spectrum in the 0.5-8.0 keV energy range (crosses) overplotted with the fitted absorbed power-law model (blue solid line) and blackbody model (red dashed line). The lower panel illustrates the ratio between the observational data and the corresponding model (blue crosses with circles for the power-law model and red crosses for the blackbody model). Right: the same figure for the new source CXOU J224257.9+444307.

Table 1

Summary of Spectral Fit Results

\begin{tabular}{|c|c|c|c|c|c|c|}
\hline \multirow{2}{*}{ Source } & \multicolumn{3}{|c|}{ Power Law } & \multicolumn{3}{|c|}{ Blackbody } \\
\hline & $\Gamma$ & $\begin{array}{c}F(0.5-8 \mathrm{keV}) \\
10^{-15}\left[\mathrm{erg} \mathrm{cm}^{-2} \mathrm{~s}^{-1}\right]\end{array}$ & $C$-statistic & $\begin{array}{c}k_{\mathrm{B}} T \\
{[\mathrm{keV}]}\end{array}$ & $\begin{array}{c}F(0.5-8 \mathrm{keV}) \\
10^{-15}\left[\mathrm{erg} \mathrm{cm}^{-2} \mathrm{~s}^{-1}\right]\end{array}$ & $C$-statistic \\
\hline MWC 656 & $2.2_{-0.9}^{+1.3}$ & $4.1_{-1.5}^{+2.3}$ & 0.3 & $0.6 \pm 0.3$ & $2.7_{-1.1}^{+1.5}$ & 3.0 \\
\hline CXOU J224257.9+444307 & $1.8 \pm 0.6$ & $8.0_{-2.1}^{+2.8}$ & 4.4 & $0.7 \pm 0.2$ & $6.0_{-1.7}^{+2.3}$ & 12.5 \\
\hline
\end{tabular}

Note. Uncertainties are at $68 \%$ c.l. Flux values are unabsorbed.

created response files in order to take into account the energydependent behavior of the instrument. We fitted the spectra with two distinct models, an absorbed power law and an absorbed blackbody, always using a fixed column density of $N_{\mathrm{H}}=1.8 \times 10^{21} \mathrm{~cm}^{-2}$ (see Munar-Adrover et al. 2014). The low number of counts required the use of the cstat statistic within XSPEC to estimate the best-fit parameters and their associated uncertainties (Cash 1979).

We show the spectra of MWC 656 and CXOU-1, together with the fitted models, in Figure 2. The results of the fits are listed in Table 1. Although both sources can be fitted with either model, the obtained $C$-statistics reveal that the power-law model is preferred since blackbody models result in an excess at both low and high energies. The obtained fluxes for the power-law models in the $0.5-8 \mathrm{keV}$ range are $4.1_{-1.5}^{+2.3} \times$ $10^{-15} \mathrm{erg} \mathrm{cm}^{-2} \mathrm{~s}^{-1}$ for MWC 656 and $8.0_{-2.1}^{+2.8} \times 10^{-15}$ erg cm $\mathrm{cm}^{-2} \mathrm{~s}^{-1}$ for CXOU-1. Although CXOU-1 appears brighter in Figure 1, the fluxes obtained by the spectral analysis are compatible at $1 \sigma$ c.l. The power-law fit for MWC 656 provides a photon index of $2.2_{-0.9}^{+1.3}$, while for CXOU-1 we obtain $1.8 \pm 0.6$, thus fully compatible at $1 \sigma$ c.l. The conclusion from the analysis of the Chandra data is that both sources have a similar spectrum, while the X-ray flux of MWC 656 is approximately half of the one measured for CXOU-1. There is no reported evidence for this source in the literature and the low number of photons prevents us from clearly establishing its nature.

We have tested if the Chandra data for the two sources produces a spectrum that needs a two-component model. We have combined the spectra of the two sources and find that the data can be reproduced with a single power law with a photon index of $2.1 \pm 0.5$ (C-statistic 3.7). Therefore, the Chandra data do not support the two-component model reported in Munar-Adrover et al. (2014).

\subsection{Radio}

MWC 656 was observed with the Karl G. Jansky Very Large Array (VLA) of the National Radio Astronomy Observatory (NRAO) on 2015 July 25 from 05:00 to 11:00 UTC (during the Chandra observation) in the A configuration. The observation was conducted at $10 \mathrm{GHz}$, with full circular polarization, using 32 spectral windows with 64 channels of $2 \mathrm{MHz}$ bandwidth each, providing a total bandwidth of $4 \mathrm{GHz}$. The amplitude calibrator was 3C 48 . The phase calibrator $\mathrm{J} 2255+4202$ was observed in 1:20-min runs interleaved with 6-min runs on the target source.

The calibration was conducted using version 4.5.0 of the CASA package ${ }^{13}$ of NRAO (McMullin et al. 2007). The data have been reduced using standard amplitude and phase calibration steps. We imaged the data using the clean procedure with a natural weighting, obtaining a synthesized beam of $0.35 \times 0.19 \operatorname{arcsec}^{2}$ in a Position Angle (P.A.) of $47^{\circ}$ (north to east). Due to the proximity of a bright quasar affecting the field of MWC 656 (see Marcote 2015), we imaged a region of a few arcmin including this quasar. We conducted a multiscale clean, considering the usual point-like components and extended components with sizes one to three times the synthesized beam (see Cornwell 2008 and Rich et al. 2008

\footnotetext{
${ }^{13}$ http://casa.nrao.edu
} 


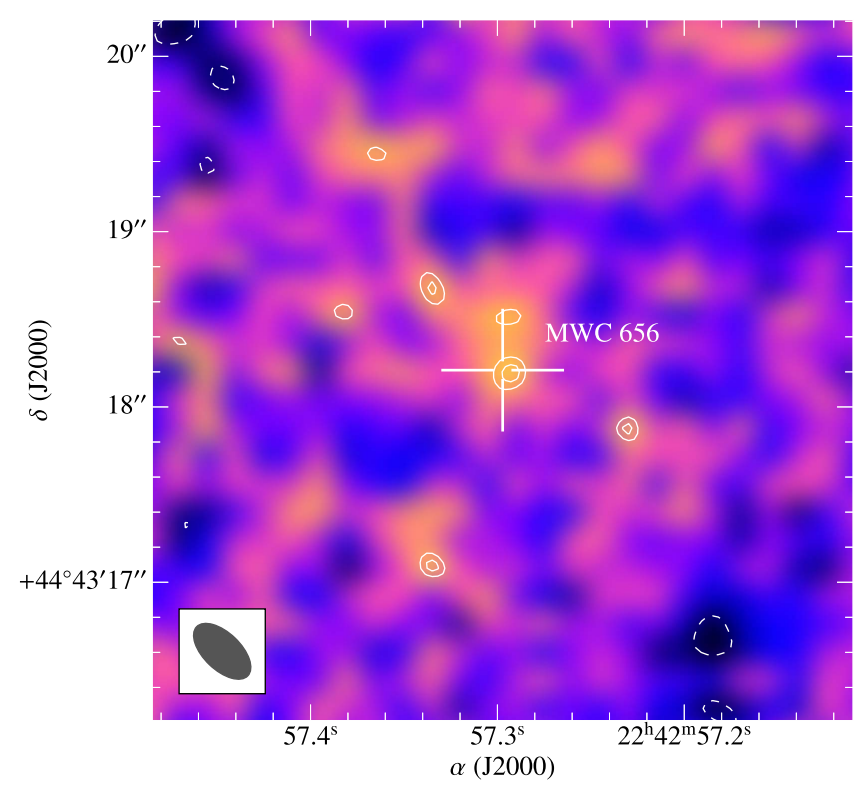

Figure 3. VLA image of $4^{\prime \prime} \times 4^{\prime \prime}$ centered at the optical position of MWC 656 (indicated by the open cross) obtained at $10 \mathrm{GHz}$ with the VLA in its A configuration. The synthesized beam size is of $0.35 \times 0.19 \operatorname{arcsec}^{2}$ in P.A. of $47^{\circ}$. Contours represent $-2.5,2.5$, and 3 times the rms noise of $1.1 \mu \mathrm{Jy}$ beam $^{-1}$.

for details). To check the reliability of the obtained results we also analyzed the data using standard clean, different weighting schemes, and different time intervals, which always led to compatible results within uncertainties.

The obtained image is shown in Figure 3. A faint radio source with a peak flux density of $3.5 \pm 1.1 \mu \mathrm{Jy}_{\text {beam }}{ }^{-1}$ is detected at the optical position of MWC 656 within uncertainties. Although this is a marginal detection, there are several facts that support the reality of the radio source as the counterpart of MWC 656: 1) it is the brightest radio source in the image, 2) it is the only one located within the Chandra $\mathrm{X}$-ray source position uncertainty, 3 ) it is fully compatible with the optical position, and 4) there is a former radio detection of MWC 656 (Dzib et al. 2015).

Inspection at the position of CXOU-1 reveals no radio counterpart, with a $3 \sigma$ flux density upper limit of $3.2 \mu \mathrm{Jy}$ for a point-like source.

\subsection{Optical}

MWC 656 was observed with different optical facilities to obtain contemporaneous photometric and spectroscopic data. Photometric observations were conducted with a passband optical filter at the $0.5 \mathrm{~m}$ robotic Telescope Fabra Roa Montsec (TFRM, Fors et al. 2013) on the nights from 2015 July 22 to 27 (except the 24), and with $B V R I_{\text {c }}$ filters at the $0.8 \mathrm{~m}$ robotic Joan Oró Telescope (TJO, Colomé et al. 2010) from 2015 July 28 onwards. Both telescopes are located at Observatori Astronòmic del Montsec (OAdM, Sant Esteve de la Sarga, Catalonia). While detailed results will be published elsewhere, during the Chandra/VLA observation MWC 656 displayed photometry compatible with its already known behavior (Paredes-Fortuny et al. 2012; Paredes-Fortuny 2016). Inspection of focused images taken on 2015 November 12 reveals no optical counterpart for CXOU-1, implying $I_{\mathrm{c}}>18.5 \mathrm{mag}$.

MWC 656 was also observed with the fiber-fed STELLA Echelle Spectrograph (SES) of the $1.2 \mathrm{~m}$ robotic STELLA-I
(ST) optical telescope (Strassmeier et al. 2004) at the Observatorio del Teide (OT, Tenerife, Spain) on the nights of 21-25 July 2015 (except the 22). The setup and the data reduction were the same as in Aleksić et al. (2015). The spectra show the presence of the double peaked He II $\lambda 4686$ emission line with an equivalent width comparable to that reported in Casares et al. (2014). We also detect other emission lines, mainly $\mathrm{H} \alpha, \mathrm{H} \beta$, and weak Fe II lines with comparable strengths to those measured by Casares et al. (2012). Therefore, MWC 656 was in a similar optical state as in past observations.

\section{Discussion and Conclusions}

The Chandra observation of MWC 656 reported here has revealed that the XMM-Newton source presented in MunarAdrover et al. (2014) was in fact the superposition of two sources: MWC 656 and the new source CXOU J224257.9 +444307 (CXOU-1). Here MWC 656 is significantly fainter than it was in the XMM-Newton observation. The VLA observation of MWC 656 has led to the detection of a faint source. This is the first simultaneous X-ray/radio detection of the first $\mathrm{Be} / \mathrm{BH}$ binary system. Here we discuss the implications of these detections.

First, it is worth noting that during the Chandra/VLA observation MWC 656 was showing optical photometric and spectroscopic properties compatible with those reported in previous works, indicating that there was no significant change in either the Be star and its circumstellar disk or the outer part of the $\mathrm{BH}$ accretion disk (He II emission line). In addition, inspection of the MAXI data (Matsuoka et al. 2009) in the direction of AGL J2241+4454 reveals no X-ray emission up to now (2009 August to 2016 October). Therefore, MWC 656 appears to have been in a long quiescent X-ray state, at least during the last 7 years.

The X-ray flux of MWC 656 provided by Chandra in 2015 July is $4.1_{-1.5}^{+2.3} \times 10^{-15} \mathrm{erg} \mathrm{cm}^{-2} \mathrm{~s}^{-1}$ in the $0.5-8 \mathrm{keV}$ energy range. For comparison, the previous X-ray observation conducted with XMM-Newton in 2013 June provided a total flux of $4.6_{-1.1}^{+1.3} \times 10^{-14} \mathrm{erg} \mathrm{cm}^{-2} \mathrm{~s}^{-1}$ in the $0.3-5.5 \mathrm{keV}$ energy range, which translates into $3.5 \pm 0.9 \times$ $10^{-14} \mathrm{erg} \mathrm{cm}^{-2} \mathrm{~s}^{-1}$ in the $0.5-8 \mathrm{keV}$ energy range. This flux is approximately one order of magnitude larger than the one measured for MWC 656 with Chandra. However, the XMMNewton source was the superposition of two sources. Assuming that CXOU-1 had a constant flux of $\sim 8.0 \times 10^{-15}$ erg $\mathrm{cm}^{-2} \mathrm{~s}^{-1}$ in the $0.5-8 \mathrm{keV}$ energy range (as determined from the Chandra data), the corresponding flux of MWC 656 at the time of the XMM-Newton observation was $\sim 2.7 \times 10^{-14} \mathrm{erg} \mathrm{cm}^{-2} \mathrm{~s}^{-1}$ in the same energy range. This would indicate a decrease of a factor of $\sim 7$ in the X-ray flux of MWC 656 between 2013 June and 2015 July.

Considering a distance of $2.6 \pm 0.6 \mathrm{kpc}$ to MWC 656 (Casares et al. 2014), the Chandra X-ray luminosity of the source in the $0.5-8 \mathrm{keV}$ energy range is $L_{\mathrm{X}}=\left(3.3_{-2.0}^{+2.4}\right) \times 10^{30}$ $\mathrm{erg} \mathrm{s}^{-1}$ (including the flux and distance uncertainties). Considering a $\mathrm{BH}$ mass in the range of 3.8-6.9 $\mathrm{M}_{\odot}$ this translates into $\left(4.9_{-3.2}^{+3.9}\right) \times 10^{-9} L_{\mathrm{Edd}}$. In the $1-10 \mathrm{keV}$ energy range these values are: $L_{\mathrm{X}}=\left(2.5_{-1.7}^{+2.6}\right) \times 10^{30} \mathrm{erg} \mathrm{s}^{-1}=\left(3.7_{-2.7}^{+4.0}\right) \times$ $10^{-9} L_{\text {Edd }}$. These luminosities and the obtained photon index are fully compatible with a $\mathrm{BH}$ in deep quiescence (Plotkin et al. 2013).

The simultaneous VLA observation of MWC 656 has yielded a detection with a peak flux density of $3.5 \pm 1.1 \mu \mathrm{Jy}$ beam $^{-1}$. 


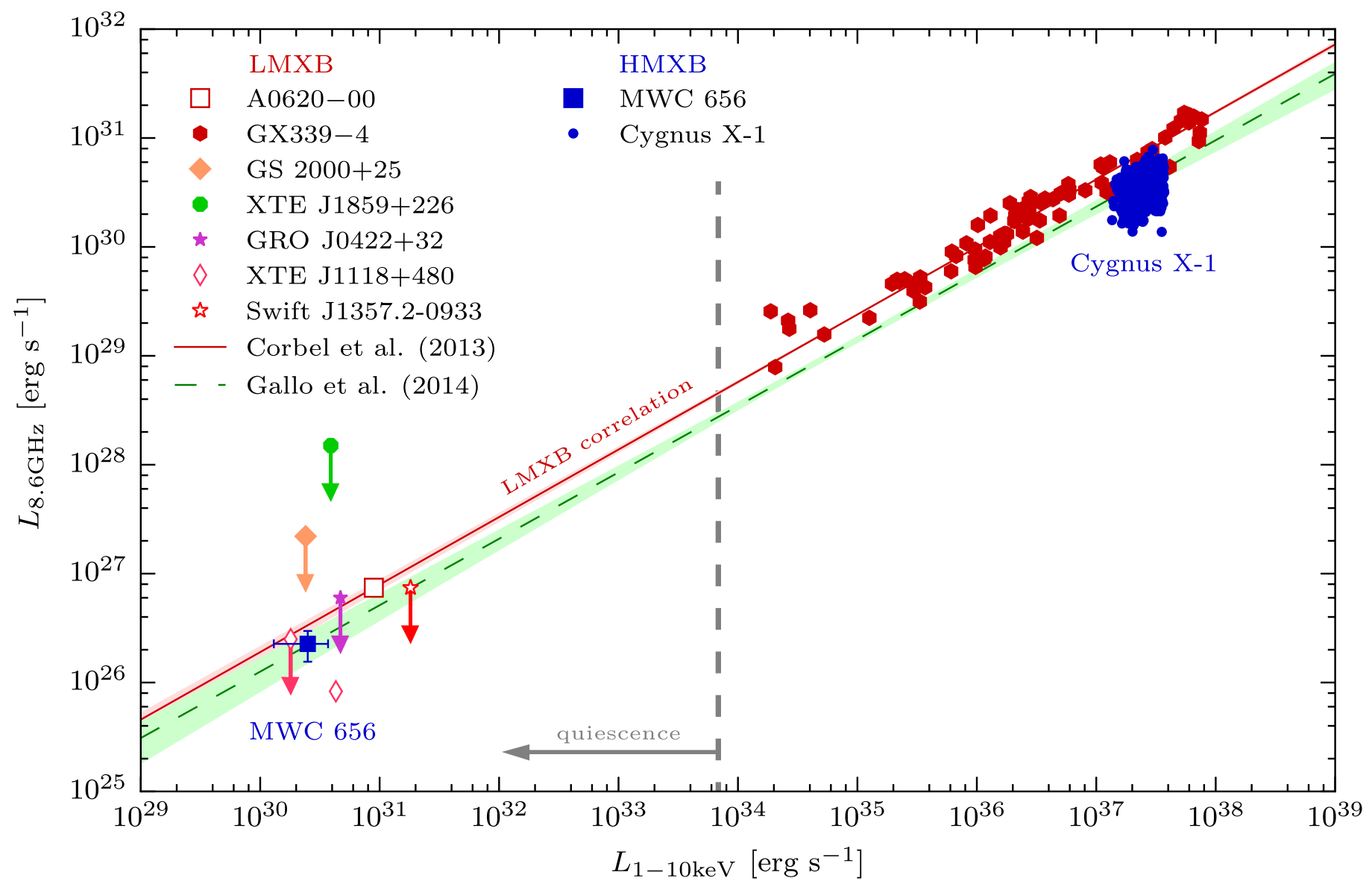

Figure 4. Radio vs. X-ray luminosity diagram including the position of MWC 656 (with $1 \sigma$ uncertainties) obtained with the Chandra/VLA observation reported in this work and the positions of a few additional significant sources from simultaneous observations. We plot the X-ray/radio correlations for BH LMXBs from Corbel et al. (2013; solid red line with a light red shadow) and from Gallo et al. (2014; green dashed line with a light green shadow). To display the luminosity range of LMXBs that follow the correlation we show data on the BH LMXBs GX 339-4 (red hexagons), as well as the two faintest sources ever detected in radio and X-rays simultaneously: A0620-00 (open red square; Gallo et al. 2006) and XTE J1118+480 (light red diamond; Gallo et al. 2014). The faintest X-ray detections of LMXBs without radio counterparts have also been plotted for reference. The small blue dots indicate the region of the parameter space where Cygnus X-1 has been detected in the low/hard state (Gallo et al. 2012). The hard-state radio-quiet outliers from the correlation have not been plotted. The gray dashed line separates the quiescent state region (left) and the other states (right) according to Plotkin et al. (2013).

The accreting BH in MWC 656 could easily produce it through synchrotron emitting electrons in a jet, as seen in many X-ray binaries (e.g., Fender \& Muñoz-Darias 2016 and references therein). In contrast, the production of such flux density by gyro-synchrotron radiation in the magnetic field of the Be star would require high magnetic fields ${ }^{14}$ combined with relatively high electron densities above the energy threshold of $10 \mathrm{keV}$ (Dulk 1985; Güdel 2002). Therefore, in what follows we consider that the detected radio emission in MWC 656 has a synchrotron origin in a jet.

We show in Figure 4 the radio versus X-ray luminosity diagram for BH X-ray binaries. The simultaneous Chandra/VLA observation of MWC 656 allows us to place the source within the diagram in a reliable way. ${ }^{15}$ MWC 656 is one of the faintest stellar-mass $\mathrm{BHs}$ ever detected in X-rays, together with

\footnotetext{
${ }^{14}$ We note the following: 1) the rapid rotation of Be stars prevents the existence of high magnetic fields in these objects; and 2) magnetism is less present in massive binaries than in isolated massive stars (Schöller et al. 2014; Neiner et al. 2015).

15 The position of MWC 656 in Figure 3 of Dzib et al. (2015) is based on their VLA data obtained in 2015 Feb-Apr and the non-simultaneous XMM-Newton data obtained in 2013 June. In addition, our Chandra observation shows that the XMM-Newton flux corresponds to two sources and that the X-ray luminosity of MWC 656 is significantly variable.
}

XTE J1118+480, GS 2000+25, XTE J1859+226, GRO J0422+ 32, A0620-00, and Swift J1357.2-0933, (Gallo et al. 2014; Miller-Jones et al. 2011; Gallo et al. 2003; Gallo et al. 2006; Plotkin et al. 2016), and the faintest one in X-rays also detected in radio. The obtained luminosities for this quiescent BH HMXB are fully compatible with those of the X-ray/radio correlations derived from quiescent BH LMXBs at the low-luminosity end. Together with Cygnus X-1 at the high-luminosity end, it is now clear, for the first time, that the accretion/ejection coupling in stellar-mass $\mathrm{BHs}$ is independent of the nature of the donor star.

Finally, given the X-ray variability found in MWC 656, future simultaneous X-ray/radio observations should allow us to trace the motion of the source in the radio versus $\mathrm{X}$-ray luminosity diagram, and directly check the slope of the correlation at the low-luminosity end for the first time in HMXBs.

We thank the anonymous reviewer for providing suggestions that helped to improve the original version of the manuscript. We thank S. Corbel for providing the data for LMXBs and E. Gotthelf for useful discussions on the Chandra astrometry. The scientific results reported in this article are based to a significant degree on observations made by the Chandra X-ray Observatory. This research has made use of software provided by the 
Chandra X-ray Center (CXC) in the application package CIAO. This research has made use of the XSPEC software. The National Radio Astronomy Observatory is a facility of the National Science Foundation operated under cooperative agreement by Associated Universities, Inc. The Common Astronomy Software Applications, CASA, is a software produced and maintained by the NRAO. The authors acknowledge support of the TFRM team for preparing and carrying out the optical photometric observations. The Joan Oró Telescope (TJO) of the Montsec Astronomical Observatory (OAdM) is owned by the Catalan Government and operated by the Institute for Space Studies of Catalonia (IEEC). This work has made use of data from the European Space Agency (ESA) mission Gaia (http:// www.cosmos.esa.int/gaia), processed by the Gaia Data Processing and Analysis Consortium (DPAC, http://www.cosmos.esa. int/web/gaia/dpac/consortium). Funding for DPAC has been provided by national institutions, in particular the institutions participating in the Gaia Multilateral Agreement. This research has made use of the MAXI data provided by RIKEN, JAXA, and the MAXI team. This research has made use of NASA's Astrophysics Data System; the SIMBAD database and the VizieR catalogue access tool (Ochsenbein et al. 2000), operated at CDS, Strasbourg, France; and Astropy, a communitydeveloped core Python package for Astronomy (Astropy Collaboration et al. 2013). We acknowledge support by the Spanish Ministerio de Economía y Competitividad (MINECO/ FEDER, UE) under grants AYA2013-47447-C3-1-P, AYA2013-47447-C3-2-P, AYA2013-42627, AYA2016-76012C3-1-P, FPA2015-69210-C6-2-R, MDM-2014-0369 of ICCUB (Unidad de Excelencia "María de Maeztu"), SEV-2015-0548 of IAC (Centro de Excelencia "Severo Ochoa"), and the Catalan DEC grant 2014 SGR 86. P.M.A. acknowledges partial support through the ASI grant No. I/028/12/0. J.C. acknowledges support by the Leverhulme Trust through the Visiting Professorship Grant VP2-2015-046.

Facilities: CXO(ACIS), VLA.

\section{References}

Aleksić, J., Ansoldi, S., Antonelli, L. A., et al. 2015, A\&A, 576, A36 Alexander, M. J., \& McSwain, M. V. 2015, MNRAS, 449, 1686 Arnaud, K. A. 1996, in ASP Conf. Ser. 101, Astronomical Data Analysis Software and Systems V, ed. G. H. Jacoby \& J. Barnes (San Francisco: CA: ASP), 17

Astropy Collaboration, Robitaille, T. P., Tollerud, E. J., et al. 2013, A\&A, 558, A33

Belczynski, K., \& Ziolkowski, J. 2009, ApJ, 707, 870

Casares, J., Ribó, M., Ribas, I., et al. 2012, MNRAS, 421, 1103

Casares, J., Negueruela, I., Ribó, M., et al. 2014, Natur, 505, 378

Cash, W. 1979, ApJ, 228, 939
Colomé, J., Ribas, I., Francisco, X., et al. 2010, AdAst, 2010, 183016

Corbel, S., Nowak, M. A., Fender, R. P., Tzioumis, A. K., \& Markoff, S. 2003, A\&A, 400, 1007

Corbel, S., Coriat, M., Brocksopp, C., et al. 2013, MNRAS, 428, 2500

Cornwell, T. J. 2008, ISTSP, 2, 793

Dulk, G. A. 1985, ARA\&A, 23, 169

Dzib, S. A., Massi, M., \& Jaron, F. 2015, A\&A, 580, L6

Fender, R. P. 2001, MNRAS, 322, 31

Fender, R., \& Belloni, T. 2004, ARA\&A, 42, 317

Fender, R. P., Belloni, T. M., \& Gallo, E. 2004, MNRAS, 355, 1105

Fender, R., \& Muñoz-Darias, T. 2016, LNP, 905, 65

Fors, O., Núñez, J., Muiños, J. L., et al. 2013, PASP, 125, 522

Fruscione, A., McDowell, J. C., Allen, G. E., et al. 2006, Proc. SPIE, 6270 $62701 \mathrm{~V}$

Gaia Collaboration, Brown, A. G. A., Vallenari, A., et al. 2016, A\&A, 595, A2 Gaia Collaboration, 2016, yCat, 1337, 0

Gallo, E., Fender, R. P., \& Pooley, G. G. 2003, MNRAS, 344, 60

Gallo, E., Fender, R. P., Miller-Jones, J. C. A., et al. 2006, MNRAS, 370, 1351

Gallo, E., Miller, B. P., \& Fender, R. 2012, MNRAS, 423, 590

Gallo, E., Miller-Jones, J. C. A., Russell, D. M., et al. 2014, MNRAS, 445, 290

Grudzinska, M., Belczynski, K., Casares, J., et al. 2015, MNRAS, 452, 2773

Güdel, M. 2002, ARA\&A, 40, 217

Lucarelli, F., Verrecchia, F., Striani, E., et al. 2010, ATel, 2761

Marcote, B. 2015, PhD thesis, Univ. de Barcelona (arXiv:1603.09276)

Markoff, S., Nowak, M., Corbel, S., Fender, R., \& Falcke, H. 2003, A\&A, 397, 645

Matsuoka, M., Kawasaki, K., Ueno, S., et al. 2009, PASJ, 61, 999

McMullin, J. P., Waters, B., Schiebel, D., Young, W., \& Golap, K. 2007, in ASP Conf. Ser. 376, Astronomical Data Analysis Software and Systems XVI, ed. R. A. Shaw, F. Hill, \& D. J. Bell (San Francisco, CA: ASP), 127

Miller-Jones, J. C. A., Jonker, P. G., Maccarone, T. J., Nelemans, G., \& Calvelo, D. E. 2011, ApJL, 739, L18

Mirabel, L. F, \& Rodríguez, I. F. 1999, ARA\&A, 37, 409

Moldón, J. 2012, PhD thesis, Univ. de Barcelona

Monet, D. G., Levine, S. E., Canzian, B., et al. 2003, AJ, 125, 984

Munar-Adrover, P., Paredes, J. M., Ribó, M., et al. 2014, ApJL, 786, L11

Munar-Adrover, P., Sabatini, S., Piano, G., et al. 2016, ApJ, 829, 101

Neiner, C., Mathis, S., Alecian, E., et al. 2015, in Proc. IAU Symp. 305, Polarimetry: From the Sun to Stars and Stellar Environments (Cambridge: Cambridge Univ. Press), 61

Ochsenbein, F., Bauer, P., \& Marcout, J. 2000, A\&AS, 143, 23

Paredes-Fortuny, X. 2016, PhD thesis, Univ. de Barcelona

Paredes-Fortuny, X., Ribó, M., Fors, O., \& Núñez, J. 2012, in AIP Conf. Proc. 1505, High Energy Gamma-Ray Astronomy (Melville, NY: AIP), 390

Plotkin, R. M., Gallo, E., \& Jonker, P. G. 2013, ApJ, 773, 59

Plotkin, R. M., Gallo, E., Jonker, P. G., et al. 2016, MNRAS, 456, 2707

Remillard, R. A., \& McClintock, J. E. 2006, ARA\&A, 44, 49

Ribó, M. 2015, in XI Multifrequency Behaviour of High Energy Cosmic Sources Workshop (MULTIF15) (Trieste: SISSA), 36

Rich, J. W., de Blok, W. J. G., Cornwell, T. J., et al. 2008, AJ, 136, 2897

Schöller, M., Hubrig, S., Briquet, M., \& Ilyin, I. 2014, in Putting A Stars into Context: Evolution, Environment, and Related Stars (Moscow: Pero), 357

Strassmeier, K. G., Granzer, T., Weber, M., et al. 2004, AN, 325, 527

Williams, S. J., Gies, D. R., Matson, R. A., et al. 2010, ApJL, 723, L93

Zdziarski, A. A. 2012, MNRAS, 422, 1750 